\title{
Review of nifedipine GITS in the treatment of high risk patients with coronary artery disease and hypertension
}

This article was published in the following Dove Press journal:

Vascular Health and Risk Management

7 May 2009

Number of times this article has been viewed

\section{Amber Lundy \\ Nahla Lutfi \\ Cherylyn Beckey}

Veterans Affairs Medical Center, Pharmacy Service, West Palm Beach, FL, USA
Correspondence: Amber Lundy, PharmD, BCPS

Veterans Affairs Medical Center

Pharmacy Service (I19)

7305 N. Military Trail

West Palm Beach, FL 33410-6400, USA

Tel + I 56I-422-5470

$\mathrm{Fax}+\mid$ 56|-422-7628

Email amber-lea.lundy@va.gov
Abstract: Nifedipine is a dihydropyridine calcium-channel blocker (CCB) introduced approximately 30 years ago for the prophylaxis of angina symptoms, and then later utilized as an anti-hypertensive agent. In the 1990s, several meta-analyses and a case-control study were published which raised concern regarding increased mortality and increased risk for myocardial infarction with short-acting nifedipine. Further evaluation of these meta-analyses and case control study underscores some important limitations and the need to further elucidate the role of this class of medications in high-risk patients. Until 2000, there was a paucity of data on the long-term effects as well as the long-term outcomes of CCBs in the treatment of stable coronary disease or in patients with manifestations of the disease such as hypertension or angina. While it has been well established that nifedipine and other dihydropyridines lower blood pressure and improve symptoms of angina, several studies were designed to evaluate the effect of dihydropyridines on "hard" outcomes, specifically cardiovascular and cerebrovascular events. In this review, we describe the clinical studies evaluating the use of nifedipine when compared to placebo as well as other anti-hypertensive therapies in an attempt to identify the most appropriate place in therapy for this class of medications and to further clarify its utilization in high-risk patients.

Keywords: nifedipine, dihydropyridine, ACTION, calcium-channel blocker, coronary heart disease, hypertension

In 2004, one of every five deaths in the United States was caused by coronary heart disease (CHD). ${ }^{1}$ It is the single largest killer of Americans, with one coronary event occurring every 26 seconds and one death every minute as a result. ${ }^{1}$ Estimated direct and indirect costs of CHD in the United States for 2008 is approximately US\$156.4 billion. ${ }^{1}$ Globally, the leading causes of death are ischemic heart disease $(12.2 \%$ of all deaths) and cerebrovascular conditions ( $9.7 \%$ of all deaths), as they caused almost $32 \%$ of all deaths in women and $27 \%$ in men in $2004 .^{2}$ Hypertension is considered one of the major risk factors in the development of cardiovascular (CV) disease as 69\% of people with first heart attack, 77\% with first stroke, and 74\% with heart failure have blood pressure higher than 140/90 mmHg. ${ }^{1}$ Thus, controlling hypertension should help lead to a reduction in morbidity and mortality. However, does blood pressure control alone help to improve cardiac outcomes, or do certain blood pressure lowering drugs innately provide more benefit than others?

In the Heart Outcomes Prevention Evaluation Study (HOPE), while ramipril induced only a modest decrease in blood pressure (by $3.3 \mathrm{mmHg} / 1.4 \mathrm{mmHg}$ ) in high-risk, mostly normotensive patients, there was a reduction in the primary endpoint 
[CV death, myocardial infarction (MI), stroke] over 4.5 years by $22 \% .{ }^{3}$ It is suggested that protective actions on left ventricular hypertrophy, endothelial function, and smooth muscle growth afforded by an angiotensin-converting enzyme inhibitor (ACE-I) help explain this phenomenon. ${ }^{4}$ However, questions arose about blood pressure reductions being underestimated since ramipril was given once daily at bedtime and blood pressure was measured during the day. Svensson et al conducted a substudy of the HOPE trial to assess the effect of ramipril on 24-hour blood pressure. ${ }^{5}$ Although office blood pressures or day ambulatory blood pressures (ABP) were not significantly reduced after 1 year, this substudy found that night and 24-hour ABP readings were significantly reduced during treatment with ramipril $10 \mathrm{mg}$ once daily at bedtime compared with placebo (by $17 \mathrm{mmHg} / 8 \mathrm{mmHg}$, p $<0.001$ and by $10 \mathrm{mmHg} / 4 \mathrm{mmHg}, \mathrm{p}=0.03$, respectively). The authors concluded that the effects seen on cardiovascular morbidity and mortality with ramipril may be related on blood pressure patterns over the 24-hour period. ${ }^{5}$

Freemantle et al conducted a systematic review of 82 randomized, controlled trials and reported a 23\% mortality reduction after long term utilization of beta-blockers (BBs) in patients that have suffered from an MI. ${ }^{6}$ Whether reducing the proarrhythmic effects of antiarrhythmic agents on board or providing anti-ischemic benefit for angina patients through their negative chronotropic and negative inotropic properties, it is recommended that BB therapy be initiated immediately in all post-MI patients and continued indefinitely for secondary prevention of MI and death..$^{7-9}$ According to the Seventh Report of the Joint National Committee on Prevention, Detection, Evaluation, and Treatment of High Blood Pressure for treatment of hypertension (JNC 7), BBs are only indicated as first-line therapy in patients with compelling indications such as stable/unstable angina, heart failure, and non-ST segment elevated MI. ${ }^{10}$ These guidelines are consistent with the 2007 guidelines for the management of hypertension from the European Society of Hypertension (ESH) and the European Society of Cardiology (ESC). ${ }^{11}$ However, with respect to primary prevention, several meta-analyses warn that BBs may not provide as much $\mathrm{CV}$ event reduction as other antihypertensives, especially in patients older than 60 years of age, and therefore are not recommended as firstline therapy for hypertension in these patients. ${ }^{12,13}$

The antihypertensive and lipid-lowering treatment to prevent heart attack trial (ALLHAT) showed that drug therapy started with a dihydropyridine (DHP) calcium channel blocker (CCB), an ACE-I, or a thiazide diuretic did not differ in the primary endpoint of combined fatal CHD and non-fatal MI. ${ }^{14}$ Given ample data for a reduction in morbidity and mortality, low cost, and relative good tolerability, thiazide diuretics are recommended as the preferred initial agent in hypertensive patients without any compelling indications. ${ }^{10}$ Beta-blockers, CCBs, ACE-I, and angiotensin II receptor blockers (ARBs) are recommended as add-on therapy if blood pressure remains elevated on thiazide monotherapy. ${ }^{10}$ The JNC 7 does recommend long-acting DHP or non-DHP CCBs if angina and blood pressure are not controlled by $\mathrm{BB}$ therapy alone, or when BBs are contraindicated. ${ }^{10}$ Although CCBs are useful in the management of angina, there is no consensus about their role in preventing $\mathrm{CV}$ events in patients with established coronary artery disease (CAD). ${ }^{15}$ The 2007 AHA Scientific Statement on treatment of hypertension in the prevention and management of ischemic heart disease takes a more comprehensive approach and recommends an ACE-I, ARB, CCB, thiazide diuretic, or a combination of those agents. ${ }^{15}$ The authors recognize the controversy over drug choice, but support this recommendation by stating that the amount of blood pressure reduction, rather than the choice of antihypertensive agent, is the major determinant in reduction of CV risk..$^{15}$ Likewise, the $2007 \mathrm{ESH} / \mathrm{ESC}$ guidelines for the management of hypertension also state that the main benefits of antihypertensive treatment are due to lowering blood pressure and are independent of the agent that is chosen. ${ }^{11}$ In addition, the ESH/ESC guidelines specify that any of the five classes of antihypertensive agents (ACE-Is, ARBs, thiazides, CCBs, or BBs) are appropriate as initial or maintenance therapy. ${ }^{11}$ Clinicians aim to provide an anti-hypertensive that will provide the most benefit with regard to reducing a patient's risk for $\mathrm{CV}$ events. Aside from pure antihypertensive effect and symptomatic improvement in angina, is there data to recommend consideration of CCBs prior to use of ACE-I or thiazides in these high-risk patients with CAD? For the purpose of this manuscript, we will focus on the use of nifedipine and also present supporting evidence of other DHP CCBs in the treatment of high-risk patients with coronary artery disease and hypertension, evaluating impact on morbidity and mortality.

Nifedipine is a DHP CCB that was introduced 30 years ago. ${ }^{16}$ It was initially developed for the prophylaxis of angina symptoms, and then later utilized as an anti-hypertensive agent. ${ }^{17}$ Nifedipine acts by inhibiting the trans-membrane influx of calcium into cardiac and vascular smooth muscle cells, thus reducing muscle contraction and has predominantly vasodilatory effects on arteries with minimal effects on the myocardium and cardiac conduction. ${ }^{16}$ The nifedipine gastrointestinal therapeutic system (GITS) tablet is 
based on an osmotic pump that releases nifedipine through a laser-drilled hole in an inert shell at a controlled rate. ${ }^{18}$ This ensures that drug release is uniform over a 24-hour period with a trough: peak ratio approaching 1 , therefore maintaining blood pressure control throughout the dosing period. This smooth reduction in blood pressure does not result in sympathetic stimulation, and there is no increase in heart rate as seen with short-acting formulations or some of the longer acting generic versions of nifedipine. ${ }^{19}$

Antiatherosclerotic effects of CCBs have been observed in animal studies and in experiments using human cultured cells. This is thought to be mediated by many factors, including antioxidant activity and enhancement of nitric oxide production. ${ }^{20}$ Most of these effects require a much higher dose than usual clinical doses, but it has been reported that some CCBs can inhibit atherosclerosis even at clinical doses. $^{21-24}$

In the 1990s, several meta-analyses and a case-control study that were published raised concern about increased mortality and increased risk for MI with short-acting nifedipine. ${ }^{25,26}$ Authors suggested that the reflex increase in sympathetic activity induced by short-acting CCBs may be the underlying mechanism of action behind observed proischemic, negative inotropic, and arrhythmogenic effects of these drugs. ${ }^{26}$ Although immediate release formulations of CCBs were approved only for treatment of vasospastic angina, they were prescribed historically to many patients as an anti-hypertensive as well. Further evaluation of these metaanalyses and case control study highlight many limitations including indication bias in the case-control study, as well as multiple inaccuracies in the Furberg analysis, which may lead to a different conclusion altogether. ${ }^{27,28}$ At present, there is no indication for short-acting CCB therapy. Long-acting CCBs have not been shown to increase mortality or risk for MI, and are commonly used anti-hypertensive medications in elderly subjects since they are well tolerated and effective. Over the past 10 years, more data demonstrating their efficacy in decreasing the incidence of $\mathrm{CV}$ events in long-term clinical trials have become available.

In 1996, Gong et al conducted a single-blind trial to assess the effectiveness of nifedipine $10 \mathrm{mg}$ twice a day (with an increase to $60 \mathrm{mg}$ daily to reach the desired blood pressure level) in elderly hypertensives over an average follow-up period of 2.5 years. ${ }^{29}$ This trial, the Shanghai trial of nifedipine in the elderly study (STONE), included 1632 patients, ages 60 to 79 years with a systolic blood pressure $(\mathrm{SBP}) \geq 160 \mathrm{mmHg}$ or diastolic blood pressure $(\mathrm{DBP}) \geq 96 \mathrm{mmHg}$. Additional medications were permissible if needed to get BP to goal (captopril 20 to $50 \mathrm{mg} /$ day and/or dihydrochlorothiazide $25 \mathrm{mg}$ /day). The endpoints of the study were combined CV events (stroke, heart failure, MI, and severe arrhythmia, and sudden death), non-CV events (malignancy, others), and all deaths. The relative risk for combined CV events was significantly reduced by $62 \%$ $(\mathrm{p}=0.0001)$, with the greatest contribution coming from reductions in risk of stroke and severe arrhythmias. There was, however, no significant difference in relative risk of death $(\mathrm{p}=0.0614)$. Overall, $65 \%$ of subjects in the nifedipine group attained the blood pressure goal of $<160 / 90 \mathrm{mmHg}$. A significant difference between the placebo group's SBP and the nifedipine group's SBP was found after only 6 months ( $p=0.0017)$, whereas the DBP difference was evident after 1 year $(p=0.0113)$. Initial assignment into the two groups (placebo and nifedipine), yielded highly comparable risk factors such as age, cholesterol, body mass index (BMI), triglycerides, glycemia, abnormalities of fundi, smoking, and alcohol consumption.

Undoubtedly, the single-blinded design is a limitation of the STONE study. In addition, 74 subjects were allowed to be reallocated from placebo to active treatment because their DBP was $\geq 110 \mathrm{mmHg}$. Another limitation could be that this study was conducted in the Chinese population where it has been demonstrated that MI is much less frequent than strokes in the People's Republic of China, ${ }^{30}$ but the authors did state that the population appeared to have predictors of clinical events (other than MI) similar to those of other ethnic groups, including the Framingham population and other Caucasians. Nifedipine retard (a long-acting formulation) significantly reduced the risk of major clinical events compared with placebo in elderly hypertensive patients in the STONE study. More recently, nifedipine retard was used in the Japan Multicenter Investigation for CV Diseases-B (JMIC-B) study and had similar efficacy to ACE-I therapy, in terms of reducing major cardiac events in patients with both hypertension and CAD. ${ }^{31}$

Data from Pristipino et al show that acetylcholine loading brought about a three-fold higher incidence of coronary spasm among Japanese patients than among Caucasian patients after MI. ${ }^{32}$ As a result, CCBs have been widely used in Japan for coronary artery disease and hypertension. In 2000, the Japanese Society of Hypertension Guidelines Subcommittee for the Management of Hypertension recommended CCBs for blood pressure control in hypertensive patients with angina pectoris, and ACE-Is in hypertensive patients with a history of MI. ${ }^{33}$ The JMIC-B compared these two agents to see which could better prevent cardiac events in 
hypertensive patients with CAD. ${ }^{31}$ This was a multi-centered prospective randomized open trial with blinded-endpoint evaluation (PROBE) that randomized 1650 patients, 75 years or younger, to 3 years of treatment with nifedipine or ACE-I. Blood pressure before antihypertensive treatment for all enrolled patients was SBP $\geq 150$ and $\mathrm{DBP} \geq 90$. Ninety-two percent of participants had previously received antihypertensive treatment and were switched to the study drug without any washout period. Exclusion criteria included DBP $\geq 120 \mathrm{mmHg}$, secondary hypertension, symptomatic cerebrovascular disease, overt heart failure, atrial fibrillation, ventricular tachycardia/ventricular fibrillation, severe renal/hepatic dysfunction, uncontrolled diabetes mellitus, and familial hypercholesterolemia. Long-acting nifedipine given at a dose of 10 to $20 \mathrm{mg}$ twice daily was compared against enalapril 5 to $10 \mathrm{mg}$, imidapril 5 to $10 \mathrm{mg}$, or lisinopril 10 to $20 \mathrm{mg}$ once daily. Treatment aimed to lower SBP to less than $150 \mathrm{mmHg}$ and DBP to less than $90 \mathrm{mmHg}$. If target not met with monotherapy, then an alpha-blocker (doxazosin, bunazosin or prazosin) was administered concomitantly. If angina persisted on monotherapy, then long-acting or short-acting nitrates and/or beta-blockers were used concomitantly.

In previously treated patients, the mean blood pressure (SBP/DBP) before starting treatment was $167 \pm 20 / 93 \pm 13 \mathrm{mmHg}$ and $165 \pm 20 / 93 \pm 13 \mathrm{mmHg}$ in the nifedipine group and ACE-I group, respectively. However, their baseline blood pressures before switching to the study drug were $146 \pm 17 / 81 \pm 11 \mathrm{mmHg}$ and $144 \pm$ $19 / 81 \pm 12 \mathrm{mmHg}$ in the nifedipine group and ACE-I group, respectively (no significant difference), while the blood pressure in the untreated patients were $160 \pm 25 / 91 \pm$ $14 \mathrm{mmHg}$ and $163 \pm 21 / 93 \pm 13 \mathrm{mmHg}$ in the nifedipine group and ACE inhibitor group, respectively (no significant difference). The fact that $92 \%$ of patients had already been taking antihypertensives (which included CCBs, ACE-Is, alpha-blockers, BBs, and diuretics) leads readers to question the strength of this study. These previously treated patients had a relatively well-controlled blood pressure before being switched to study drugs and there was no mention of average duration of treatment prior to initiation of study drugs. It is reasonable to question the effect of this study design on the results of the study.

At the end of treatment, the mean dose of nifedipine was $31.9 \pm 10.7 \mathrm{mg} / \mathrm{day}$, while that of enalapril was $5.6 \pm 2.5 \mathrm{mg} /$ day, lisinopril was $10.2 \pm 3.9 \mathrm{mg} / \mathrm{day}$, and imidapril was $6.8 \pm 2.4 \mathrm{mg} / \mathrm{day}$. The mean reduction of blood pressure (SBP/DBP) was $11 / 5 \mathrm{mmHg}$ in the nifedipine group and 7/4 mmHg in the ACE-I group ( $p<0.01)$. The percentage of patients who received concomitant therapy with ACE-I, nitrates, and BBs were equivalent in both treatment groups. However, the difference in rate of alpha-blocker was statistically significant $(6.3 \%$ in CCB group and $10.7 \%$ in ACE-I group, $\mathrm{p}<0.0012$ ). A subgroup analysis showed that alpha-blocker use had no significant impact on incidence of cardiac events. The primary endpoint was the overall incidence of cardiac events (cardiac death or sudden death, MI, hospitalization for angina pectoris or heart failure, serious arrhythmia, and coronary interventions). The primary endpoint occurred in 116 patients (14.0\%) in the nifedipine retard group and 106 patients $(12.9 \%)$ in the ACE-I group. There was no significant difference between the two groups $(\mathrm{p}=0.75)$.

In the CCB group, patients with a history of MI had a reduction in the risk of hospitalization for angina pectoris of $58 \%$ when compared with the ACE-I group. The antispastic effect of this drug on the coronary arteries may likely be a factor leading to the reduced incidence of hospitalization for angina pectoris in post-MI patients. Authors highlight that baseline and end of study blood pressure of patients in the JMIC-B study were comparable to those of patients in ALLHAT. Blood pressure was well-controlled in both trials, and may explain consistency in results between JMIC-B and ALLHAT. ${ }^{14,31}$ Upon review of dosage regimens used in this study, the doses seemed inadequate when compared to doses used in Americans and Europeans. However, these dose levels are considered optimum for efficacy and safety in Japanese patients, and standard doses of these ACE-Is were determined in multi-center clinical studies based on the Japanese guidelines for evaluating antihypertensive drugs. ${ }^{34-37}$ The effects of nifedipine on the progression of coronary atherosclerosis were compared with those of ACE-Is using quantitative coronary angiogram (QCA) of the CV measurement system in a JMIC-B side arm study. ${ }^{38}$ Seventy-seven institutions specializing in CV diseases agreed to submit coronary angiogram films of patients to the core angiographic laboratory before and 36 months after the start of treatment. Study analyses were performed on the data from 83 and 79 patients in the nifedipine and ACE-I groups, respectively. These analyses showed that nifedipine was better than ACE-Is in inhibiting the progression of coronary atherosclerosis and in the reduction of development of new lesions. Despite the effects on atherosclerosis based on QCA, clinically this did not translate into a reduction of morbidity and there were no significant differences that were found in the incidence of cardiac events between the groups (nifedipine 25 patients; ACE-I 22 patients). 
In recent years, a couple of key clinical trials have expanded the body of evidence available on modified-release formulations of nifedipine. The International Nifedipine GITS study: Intervention as a Goal in Hypertension Treatment (INSIGHT) was the first, large, double-blind, randomized trial undertaken exclusively in high-risk hypertensive patients, with $\mathrm{CV}$ events as a prospectively defined primary end-point. ${ }^{39}$ This trial compared the effects of nifedipine GITS $30 \mathrm{mg}$ daily with co-amilozide $25 / 2.5 \mathrm{mg}$ daily (combination of hydrochlorothiazide and amiloride) on CV mortality and morbidity in 6321 patients aged 55 to 80 years old from Europe and Israel. This study consisted of hypertensive patients (blood pressure $\geq 150 / 95 \mathrm{mmHg}$, or $\mathrm{SBP} \geq 160 \mathrm{mmHg}$ ) with an additional risk factor which included hyperlipidemia, smoker, family history of MI in parent or sibling $<50$ years old, left ventricular hypertrophy, CHD, left ventricular strain, peripheral vascular disease, or proteinuria. Dose doubling and additional therapy with atenolol $25 \mathrm{mg}$ daily or enalapril $5 \mathrm{mg}$ daily was allowed if BP was $>140 / 90 \mathrm{mmHg}$ after 2 weeks of treatment.

The primary outcome (CV death, MI, heart failure, and death) occurred in 200 (6.3\%) patients in the nifedipine group compared to $182(5.8 \%)$ in the co-amilozide group. These findings were not significant $(\mathrm{p}=0.35)$ and showed that once daily nifedipine was equally effective as co-amilozide in preventing overall $\mathrm{CV}$ or cerebrovascular complications over a mean follow-up of 4 years. Mean BP fell by $33 / 17 \mathrm{mmHg}$ and remained close to $138 / 82 \mathrm{mmHg}$ in both groups (also no difference between the groups). Demography and distribution of risk factors did not differ significantly between the treatment groups. Event rates were higher in some risk groups than others and most did significantly affect outcomes (smoking, previous MI, proteinuria, sex, and age), but there were no apparent differences between the two treatment groups. The authors reported 1259 patients received atenolol and 756 patients received enalapril as add on treatment, with a similar fall in blood pressure, but neglected to mention any possible influence that these additional drugs may have had on the outcome. When considering vascular function, substudies of INSIGHT showed that nifedipine GITS compared with diuretic therapy, slowed the progression of carotid intimamedia thickening and coronary calcification, and improved endothelial function in patients with $\mathrm{CV}$ disease. ${ }^{40}$ However, this has yet to be demonstrated to be clinically important.

Clinical trials such as INSIGHT have indicated that nifedipine GITS significantly lowers blood pressure while reducing $\mathrm{CV}$ risk, in addition to being effective and safe in a broad spectrum of patients. Similar results were observed with amlodipine in the ALLHAT trial which showed a lower incidence of stroke, combined CV disease, angina and peripheral arterial disease when compared with lisinopril. ${ }^{14}$ Nifedipine and other DHP CCBs have also been studied extensively in the treatment of angina pectoris. The majority of these studies were designed to assess symptomatic improvement defined as a reduction in angina symptoms. However, until 2004, there was a paucity of data about the long-term effects as well as the long-term outcomes of CCBs in the treatment of stable coronary disease or in patients with manifestations of the disease such as hypertension or angina.

A coronary disease trial investigating outcome with nifedipine GITS (ACTION) was one of the largest studies designed to evaluate the effect of long-acting nifedipine GITS on mortality and CV morbidity in patients with stable angina requiring treatment. ${ }^{41}$ This was a randomized, placebo controlled, double-blinded trial, (planned duration 4.5 to 6 years) in which 6084 men and 581 women were recruited by 291 centers from 19 countries. Patients were eligible for the study if they were 35 years of age and older, had stable angina pectoris for at least one month, required therapy to prevent symptoms, and either had a history of MI, CAD confirmed by angiography, or had a positive exercise test or perfusion defect. Exclusion criteria consisted of orthostatic hypotension (or DBP $\leq 90 \mathrm{mmHg}$ ), $\mathrm{SBP} \geq 200 \mathrm{mmHg}$ or DBP $\geq 105 \mathrm{mmHg}$, and patients with left ventricular ejection fraction $<40 \%$. Patients were randomly assigned to nifedipine GITS $(\mathrm{n}=3825) 30$ to $60 \mathrm{mg}$ daily or matched placebo $(n=3840)$ in addition to the basic regimen the patient was taking. During the study, providers were permitted to treat symptomatic patients with conventional drugs. The primary efficacy outcome was major $\mathrm{CV}$ event-free survival defined as death from any cause, acute MI, refractory angina, new overt heart failure, debilitating stroke, and peripheral revascularization. The primary combined safety outcome included death from any cause, acute MI, and debilitating stroke. Secondary outcomes included any CV event, any death, any CV event or procedure, and any vascular event or procedure. The mean follow up of this trial was 4.9 years. Mean baseline blood pressure measurement in both groups was approximately $137 / 80 \mathrm{mmHg}$. However, $>50 \%$ of the population has a blood pressure $\geq 140 / 90$ at baseline.

There was no statistically significant difference with regard to the primary efficacy outcome between nifedipine GITS and placebo (hazard ratio 0.97 [95\% CI 0.88-1.07], $\mathrm{p}=0.54)$. When the incidence of clinical events was evaluated separately, new overt heart failure was the only 
component of the primary endpoint that reached statistical significance favoring nifedipine (29\% reduction, $\mathrm{p}=0.015$ ). However, nifedipine GITS did show a benefit with regard to a reduction in the need for coronary procedures and symptom interventions. Coronary angiography was reduced by $18 \%(\mathrm{p}<0.0001)$ and coronary artery bypass grafting was reduced by $21 \%(\mathrm{p}=0.002)$. There were no statistically significant differences in the rates of MI between nifedipine GITS compared to placebo (hazard ratio 1.04 [95\% CI $0.88-1.24], \mathrm{p}=0.62$ ), and this information, in part, dispels past controversies pertaining to an increased risk of MI with other formulations of nifedipine.

While it is well established that a reduction in blood pressure translates to a reduction in $\mathrm{CV}$ events, it is of interest that despite a mean reduction in a blood pressure of 3.9/2.4 mmHg in normotensive patients in the ACTION trial, this did not translate to a reduction in the primary outcome. A large percentage of patients in the ACTION trial were receiving medications that are well established in risk reduction such as statins, $\mathrm{BBs}$, and aspirin. This potentially makes additional benefit more difficult to achieve.

However, in the ACTION trial, $52 \%$ of patients were hypertensive at baseline (defined as blood pressure of $\geq 140 / 90$ ). Keeping in mind that some patients designated as "normotensive" at baseline, quite possibly had controlled hypertension as this definition was irrespective of treatment. In the predefined subgroup of patients with hypertension, there was a statistically significant reduction of $13 \%$ in the primary combined endpoint of death from any cause, acute MI, refractory angina, new overt heart failure, debilitating stroke, and peripheral revascularization. However, when evaluated separately, new overt heart failure and debilitating stroke were the only components to reach statistical significance. Therefore, in patients with CAD and hypertension, nifedipine GITS may be beneficial as add-on therapy as the design of ACTION did not include a washout period, and as previously mentioned a high percentage of patients were already receiving therapies proven to reduce $\mathrm{CV}$ risk.

The INSIGHT and ACTION trials, along with the STONE and JMIC-B trial, provide evidence that long acting nifedipine can be used safely in high-risk, elderly patients to lower blood pressure and possibly improve clinical outcomes (Table 1). ${ }^{29,31,39,41}$ Nifedipine has not been proven inferior to other therapies and in fact may provide benefit with regard to reductions in hospitalizations as well as $\mathrm{CV}$ procedures. However, the role of nifedipine GITS as first-line therapy or as monotherapy in high-risk patients needs to be further elucidated.
The above trials examined the use of nifedipine and its impact on the risk of CV events, however one must also consider clinical trials involving other DHP CCBs, as they may provide more insight for use of this agent in the treatment of high risk patients with CAD and hypertension. The question of whether DHP CCBs have benefit beyond blood pressure lowering, possible benefit due to anti-anginal properties, and benefit when added to standard therapy in patients with CAD has been addressed in multiple clinical trials. In the prospective randomized evaluation of the vascular effects of the Norvasc (PREVENT) study, the effect of amlodipine on progression of coronary atherosclerosis was compared to placebo in 825 patients over 36 months. ${ }^{42}$ There was no statistically significant difference in major $\mathrm{CV}$ event rates and in the average 36-month reductions in the minimal lumen diameter (0.084 versus $0.095 \mathrm{~mm} ; \mathrm{p}=0.38)$. Results of the PREVENT trial differ from that of the JMIC-B Side Arm Study, which did show a suppression of the progression of coronary atherosclerosis. ${ }^{38}$ Shinoda et al noted the difference in study population and emphasize that Japanese patients with atherosclerosis have a higher incidence of coronary spasm which could aggravate atherosclerosis, thereby explaining the beneficial effects of $\mathrm{CCBs}$ on plaque rupture and erosion. ${ }^{32,43}$ The NICOLE study evaluated the effect of nisoldipine on progression of coronary atherosclerosis. ${ }^{44}$ There was no statistically significant difference when compared to placebo. In the CAMELOT trial, there was a statistically significant reduction in coronary revascularizations with amlodipine. ${ }^{45}$ However, similar to the CAMELOT trial, results of the PREVENT and NICOLE studies led to fewer hospitalizations for unstable angina and revascularizations. Perhaps the possibility exists of improved symptom control leading to a reduction in patients seeking medical care, and hence hospitalizations and procedures.

However, there are some trials that raised questions regarding the impact of DHP CCBs on reduction of $\mathrm{CV}$ events. The fosinopril versus amlodipine cardiovascular events randomized trial in patients with hypertension and non-insulin dependent diabetes mellitus (FACET) was an open-labeled trial that randomized 380 hypertensive diabetics to fosinopril $20 \mathrm{mg} /$ day or amlodipine $10 \mathrm{mg} /$ day and followed for up to 3.5 years. ${ }^{46}$ By the end of the study, the SBP was $4 \mathrm{mmHg}$ lower in the amlodipine group compared with fosinopril ( $\mathrm{p}<0.01)$, while both groups had the same reduction in DBP compared with baseline ( $8 \mathrm{mmHg}$ ). Despite a significantly greater reduction in SBP in the CCB group, the proportion of patients diagnosed with the combined secondary end point of stroke, acute MI, or hospitalized 


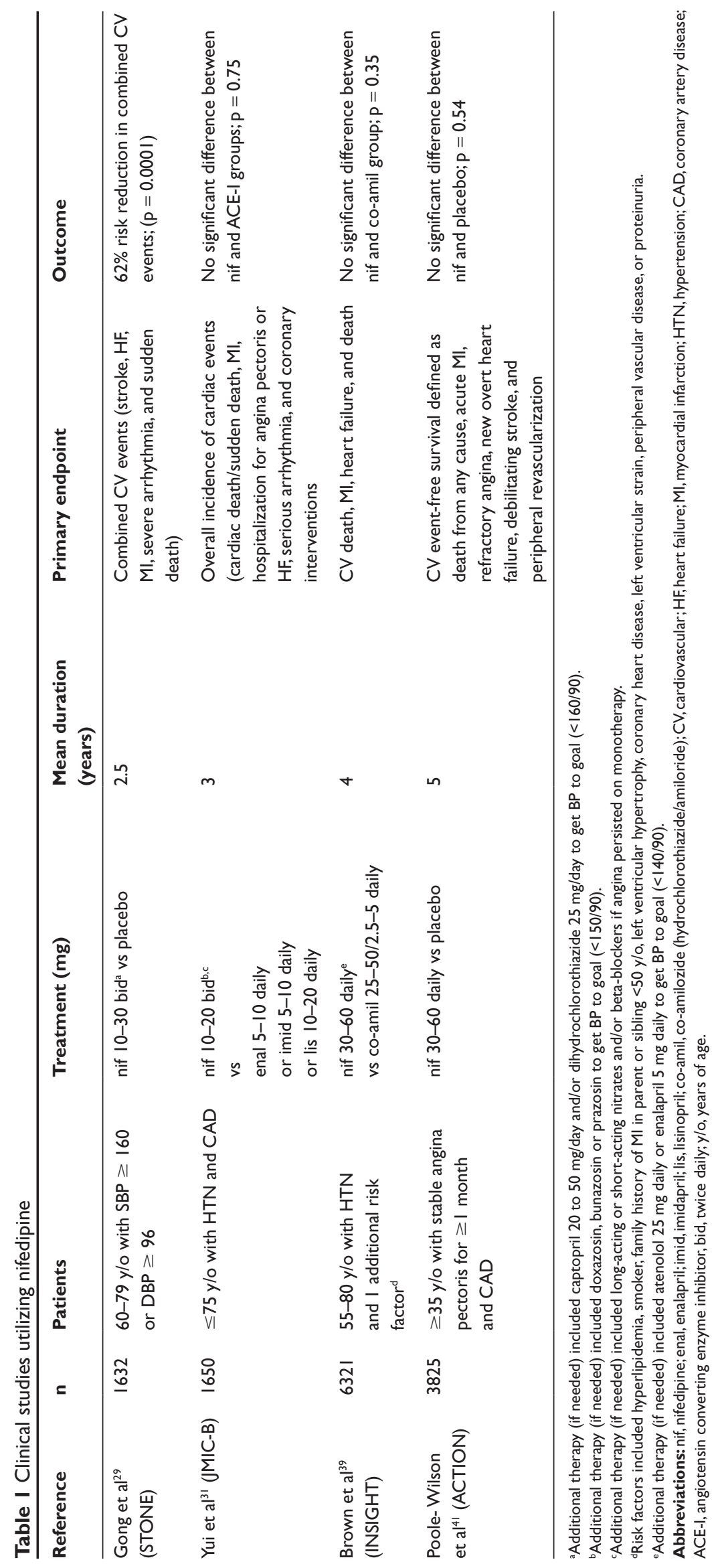


angina was significantly lower in the fosinopril group compared with amlodipine group $(\mathrm{p}=0.030, \mathrm{HR}=0.49$, $95 \% \mathrm{CI}=0.26-0.95)$. These results raised doubts and highlighted the limitations of blood pressure lowering as a surrogate marker of clinical efficacy of antihypertensive therapy. However, limitations of this trial should be pointed out. For example, the timing of blood pressure measurement was likely to be close to the peak effect of amlodipine and to the trough effect of fosinopril possibly leading to greater reduction in SBP found in the $\mathrm{CCB}$ group than with the ACE-I group. ${ }^{47,48}$ In addition, Tatti suggests that the relative dose of amlodipine was greater than that of fosinopril, or that amlodipine was more effective than fosinopril in lowering blood pressure. ${ }^{46}$ It is also important to report that almost one third of the patients used combination treatment of fosinopril plus amlodipine, and as a result, in the intention-to-treat analyses, any treatment effect would be diluted. Lastly, this trial was open-label, conducted at a single site, and was not designed and powered to assess a difference between the two treatments in vascular events.

The results of FACET are in agreement with those of the Multicenter Isradipine Diuretic Atherosclerosis Study (MIDAS). In both trials, hypertensive patients with diabetes or impaired glucose metabolism who received alternative treatments had a significantly lower risk of $\mathrm{CV}$ events, compared with those who received a CCB. Results of animal studies have demonstrated the antiatherogenic properties of isradipine as well as other CCBs. ${ }^{49-53}$ MIDAS compared the rate of progression of mean maximum intimal-medial thickness (IMT) in carotid arteries during antihypertensive therapy with isradipine versus hydrochlorothiazide over 3 years. ${ }^{54}$ By 6 months, DBP in both groups was reduced by about $13 \mathrm{~mm} \mathrm{Hg}$ from baseline, whereas SBP was reduced by $19.5 \mathrm{mmHg}$ in the hydrochlorothiazide group and $16.0 \mathrm{mmHg}$ in the isradipine group $(p=0.002)$. No difference was seen in the rate of progression of IMT between the two treatment groups over 3 years $(p=0.68)$. However, there was a significant increase in non-major vascular events and procedures (transient ischemic attack, dysrhythmia, aortic valve replacement, and femoral popliteal bypass graft) in the isradipine group $(n=40$; $9.05 \%)$ versus the hydrochlorothiazide group $(n=23 ; 5.22 \%)$ $(\mathrm{p}=0.02)$. Moreover, at the final clinic visit, $25 \%$ of the isradipine group and $28 \%$ of the hydrochlorothiazide group were taking enalapril as blood pressure was uncontrolled on monotherapy. Given that almost one-third of each group was on ACE-I therapy, this introduces a confounder and one must evaluate the results of this study in proper context. One must also notice that the rate of progression of IMT observed in MIDAS (0.03 mm/year) was much slower than the rate observed in a small pilot study of 28 untreated hyperlipidemic patients used to calculate the sample size of MIDAS $(0.15-0.20 \mathrm{~mm} /$ year $){ }^{55,56}$ This suggests that IMT progression rate may differ in different populations, and lowering blood pressure is not the only answer in reducing CV events. It must be part of a strategic plan which includes reduction of cholesterol, diabetes control, and healthy lifestyle void of tobacco and full of physical activity and a healthy diet.

There were multiple studies that looked at DHP CCBs compared to ACE-Is, diuretics, and $\mathrm{BBs}$, and provided evidence that DHP CCBs had similar antihypertensive efficacies and CV event rates similar to these classes. The Swedish Trial in Old Patients with Hypertension-2 study (STOP-2) compared the effects of conventional therapy (atenolol $50 \mathrm{mg}$, metoprolol $100 \mathrm{mg}$, pindolol $5 \mathrm{mg}$, or hydrochlorothiazide $25 \mathrm{mg}$ plus amiloride $2.5 \mathrm{mg}$ daily) with that of newer agents (enalapril $10 \mathrm{mg}$ or lisinopril $10 \mathrm{mg}$, or felodipine $2.5 \mathrm{mg}$ or isradipine $2-5 \mathrm{mg}$ daily) on CV mortality and morbidity in elderly patients. ${ }^{57}$ This trial showed that there were no differences in blood pressure, CV mortality, or major events between all the study groups after 4 years of follow-up. The primary combined endpoint of fatal stroke, fatal MI, and other fatal CV disease occurred in 221 of 2213 patients in the conventional (19.8 events per 1000 patient years) and in 438 of 4401 in newer drugs group (19.8 per 1000 patient years); $\mathrm{p}=0.89$. STOP-2 results suggested that newer antihypertensive drugs were equally efficacious and the choice of treatment will, therefore, be related to other factors such as cost, side-effects, and co-existing conditions.

Unlike STOP-2, the ASCOT-BPLA provided evidence on the contrary, and showed a DHP CCB to have better antihypertensive efficacy and greater reduction in $\mathrm{CV}$ event rates when compared to other agents. The Anglo-Scandinavian cardiac outcomes trial-blood pressure lowering arm (ASCOT-BPLA) was a multi-centered, prospective, randomized controlled trial that compared the incidence non-fatal $\mathrm{MI}$ and fatal CHD in patients on amlodipine 5 to $10 \mathrm{mg}$ plus perindopril 4 to $8 \mathrm{mg}$ versus atenolol 50 to $100 \mathrm{mg}$ plus bendroflumethiazide 1.25 to $2.5 \mathrm{mg}$ daily. ${ }^{58}$ This trial included patients who were mostly white men with mean age of 63 years old. These patients had either treated $(>80 \%)$ or untreated hypertension with an average blood pressure of 164/95 $\mathrm{mmHg}$ and at least three other cardiovascular risk factors which included left ventricular hypertrophy, type 2 diabetes, peripheral arterial disease, previous stroke or transient ischemic attack, male sex, age 55 and older, 
microalbuminuria or proteinuria, smoking, ratio of total cholesterol to high-density lipoprotein cholesterol (HDL) of 6 or higher, and family history of premature CHD. Patients were randomized to amlodipine adding perindopril as required to reach blood pressure targets or atenolol adding bendroflumethiazide and potassium as required. At each follow-up visit, drug therapy was increased to achieve target blood pressure of $<140 / 90$ for non-diabetics and $<130 / 80$ for diabetic patients.

Blood pressures were lower throughout the trial in the amlodipine-based regimen compared with those on the atenolol-based regimen, with an average difference of 2.7/1.9 mmHg. By the end of the trial, blood pressure readings fell to $136.1 \pm 15.4 / 77.4 \pm 9.5 \mathrm{mmHg}$ and $137.7 \pm 17.9 / 79.2 \pm 10 \mathrm{mmHg}$ in the amlodipine and atenolol-based regimens, respectively. It is important to note that by the end of the study, most patients were taking at least two antihypertensive agents (78\%), with only $15 \%$ and $9 \%$ taking amlodipine and atenolol monotherapy, respectively. There was no statistically significant difference in primary endpoint of non-fatal MI plus fatal CHD when comparing both groups, however there were significant reductions in most secondary endpoints which included fatal and non-fatal stroke (327 versus $422 ; p=0.0003$ ), total cardiovascular events and procedures (1362 versus 1602; $\mathrm{p}<0.0001$ ), and all-cause mortality (738 versus $820 ; \mathrm{p}=0.025$ ). The trial was stopped early as those in the atenolol-based group had significantly higher mortality and worse outcomes on several secondary endpoints as mentioned above. Authors noted that this study was powered for 1150 patients to reach primary endpoint, however only 903 patients actually had such events because of early termination. Therefore, ASCOT was underpowered for the primary endpoint.

In the post-hoc analyses, which combined the primary endpoint plus coronary revascularizations, the event rate for amlodipine-based regimen was significantly reduced by $14 \%$ when compared to atenolol-based regimen $(\mathrm{p}=0.0058)$. For justification of this combined endpoint, authors acknowledge the increase in use of interventional procedures to prevent future coronary events in the management of $\mathrm{CHD}$, which has become routine clinical practice since the design of this trial. It was mentioned that possible explanations for differences in outcome in treatment groups may include higher body mass index, triglyceride levels, creatinine concentrations, and fasting blood glucose values, as well as a lower HDL in atenolol-based regimen group, which was found at final visit when compared to amlodipine-based regimen group. It is also important to note that about $40 \%$ of patients used antihypertensive agents other than those outlined in this study, and $8 \%$ were on four or more drugs. This study's results reinforce the notion that most hypertensive patients need at least two drugs to reach target blood pressure. Authors concluded that the combination of a $\mathrm{BB}$ and diuretic should not be recommended over CCB/ACE-I for routine use, as the $\mathrm{CCB} / \mathrm{ACE}-\mathrm{I}$ drug regimen proved better in reduction of cardiovascular events and all-cause mortality.

The Systolic Hypertension in Europe Trial (Syst-Eur) examined whether antihypertensive therapy could reduce $\mathrm{CV}$ complications of isolated systolic hypertension. ${ }^{59}$ Active treatment started with nitrendipine, and if necessary, this drug was combined with or replaced by enalapril (up to $20 \mathrm{mg}$ daily), hydrochlorothiazide (up to $25 \mathrm{mg}$ daily), or both. Active treatment reduced occurrence of all strokes by $42 \%(p=0.003)$, decreased all fatal and non-fatal cardiac endpoints, including sudden death, by $26 \%(\mathrm{p}=0.03)$, and reduced all fatal and non-fatal CV endpoints by $31 \%$ $(p<0.001)$. Unlike the FACET or the national intervention cooperative study in elderly hypertensives study (NICS), the Syst-Eur Trial included patients that were not necessarily older, but sicker, as in the two treatment groups combined, 29.9\% (1402) of patients had CV complications at randomization. Forty-one percent (575) and 7.3\% (103) of these patients had CHD and cerebrovascular disease, respectively. ${ }^{46,59,60}$ The absolute number of strokes prevented by active treatment was similar to that of the STOP (Swedish trial in old patients with hypertension) trial, and the results for stroke and MI were similar to that of the SHEP (systolic hypertension in the elderly program) trial. ${ }^{61,62}$ At the time of this trial, diuretics and BBs were the only antihypertensives used in long term clinical trials and were shown to reduce morbidity and mortality. This study provided some evidence that newer agents (CCBs and ACE-Is) also played a part in reducing $\mathrm{CV}$ complications. One may question the protective effects of CCBs alone, given that ACE-I and/or thiazide was added for blood pressure lowering. In response to this question, the Syst-Eur trial reports that the benefit of active treatment was seen soon after randomization when most patients were still on CCB monotherapy. ${ }^{59}$

Diuretics are recommended first line for the treatment of hypertension, however there has been a decrease in diuretic use and an increase in CCB use over the years. NICS aimed to compare the effects of a diuretic and a CCB in preventing $\mathrm{CV}$ complications in elderly hypertensive patients in Japan. ${ }^{60}$ The total number of $\mathrm{CV}$ complications during the 5 -year period was $21(10.3 \%)$ in the nicardipine group and $18(8.6 \%)$ in the diuretic group $(\mathrm{p}=0.923)$. This trial showed that nicardipine 
was equivalent to trichlormethiazide in its effect against $\mathrm{CV}$ complications in elderly patients with hypertension. The NICS study was the first to make a direct comparison of a DHP CCB and a diuretic. Unlike SHEP, STOP, STONE, or Syst-EUR trial, this study was not a placebo-controlled trial, as a placebo comparison was ruled out by the steering committee because of ethical considerations based on the known efficacy of diuretics..$^{29,59-62}$ In the MIDAS study, there was a trend toward increased incidence of vascular events in patients treated with CCB compared with diuretic $(5.65 \%$ vs $3.17 \%$, respectively; $\mathrm{p}=0.07) .{ }^{54}$ Authors suggest that reasons contributing to the difference between these results and those of the NICS study may be the greater decrease in SBP in the diuretic group versus isradipine group in MIDAS (19.5 $\mathrm{mmHg}$ vs $16 \mathrm{mmHg}$, respectively; $\mathrm{p}=0.002$ ).

Given the fact that this study was conducted in Japan, one may question the population validity of these results. One may also question the direct comparison between these agents and the incidence of $\mathrm{CV}$ complications given that these complications are affected by patient age, sex, blood pressure, and atherosclerotic complications before the study. However, the CV complication rate per 1000 persons per year in the treated groups was 21 in the MRC (medical research council trial of treatment of hypertension in older adults) study, 33.5 in the STOP study, 23.3 in the Syst-Eur study, 21.4 in the Syst-China study, and 27.8 in the nicardipine group and 26.8 in the diuretic group in the NICS study. ${ }^{59-61,63,64}$ The similarities with the values from these previous studies suggest that the incidence of CV complications in Japan is not different from that in Europe, the United States, or China.

The Avoiding Cardiovascular Events through Combination Therapy in Patients Living with Systolic Hypertension (ACCOMPLISH) trial was a double-blind trial that randomized 11,506 patients with hypertension who were at high risk for $\mathrm{CV}$ events to receive treatment with either benazepril plus amlodipine or benazepril plus hydrochlorothiazide. ${ }^{65}$ The primary end point was the composite of death from CV causes, nonfatal MI, non-fatal stroke, hospitalization for angina, resuscitation after sudden cardiac arrest, and coronary revascularization. The average blood pressures after dose adjustment were $131.6 / 73.3 \mathrm{mmHg}$ in the benazepril-amlodipine group and $132.5 / 74.4 \mathrm{mmHg}$ in the benazepril-hydrochlorothiazide group. There were 552 primary-outcome events in the benazepril-amlodipine group $(9.6 \%)$ and 679 in the benazepril-hydrochlorothiazide group (11.8\%). This represented an absolute risk reduction of $2.2 \%$ with benazepril-amlodipine and a relative risk reduction of 19.6\% (95\% confidence interval [CI]: 0.72-0.90; $<<0.001$ ). Authors discuss the superiority of amlodipine when compared to hydrochlorothiazide in reducing $\mathrm{CV}$ events in this trial. This differs from ALLHAT results, which showed no difference in event rate when comparing $\mathrm{CCB}$ and thiazide diuretic. Critics suggest that the difference in outcomes may be due to the fact that ALLHAT used chlorthalidone, which has double the potency and a longer duration of action compared to hydrochlorothiazide. ${ }^{66}$ It is also noted that the combination of $\mathrm{CCB}$ with a drug that inhibits the reninangiotensin system may offer unique benefits when compared to the CCB monotherapy that was utilized in ALLHAT. ${ }^{66}$

Hosoda et al report that the risk of death in patients with CAD receiving secondary prevention therapy is 20 times as high as that in healthy individuals. ${ }^{67}$ Clinicians utilize statins, aspirin, and antihypertensives to alleviate this risk of death and reduce morbidity in efforts to sustain a reasonably good quality of life. In this review, we are charged with investigating nifedipine GITS and its role in the treatment of hypertension in high risk patients with CAD. Four major studies (INSIGHT, ACTION, STONE, JMIC-B) that evaluated the use of nifedipine concluded that nifedipine GITS is not inferior to other antihypertensives in reducing CV events (Table 1). ${ }^{29,31,39,41}$ However, when compared to diuretics and ACE-Is, nifedipine slowed the progression of coronary atherosclerosis and reduced the development of new lesions. Yet, clinically this did not translate into a reduction of morbidity and mortality. More importantly, we recognize the fewer hospitalizations for unstable angina and revascularizations given nifedipine's potential for improved symptom control leading to a reduction in patients seeking medical care, and hence hospitalizations and procedures. The review of trials that included other DHP CCBs, lead to the same conclusion of non-inferiority of DHP CCBs. In most of the studies presented in this review, the trials were designed to test single agents. However, other drug treatments were often added for blood-pressure control, thus creating confounders and changing the interpretation of the effects of the study drug on end points. ${ }^{65}$ Based on the results of these studies, nifedipine GITS may be recommended as add-on therapy in patients with CAD to help lower blood pressure and possibly improve $\mathrm{CV}$ risk. Given that most patients with hypertension will require two or more agents for control, nifedipine GITS would be an appropriate choice in combination therapy. However, the role of nifedipine GITS as first-line therapy or as monotherapy in high-risk patients needs to be further examined. 


\section{Disclosures}

The authors disclose no conflicts of interest.

\section{References}

1. American Heart Association. Heart Disease and Stroke Statistics - 2008 Update. Dallas, Texas: American Heart Association; 2008.

2. Mathers C, Boerma T, Fat DM; for WHO staff. The global burden of disease: 2004 update. Accessed Nov 2008. Available from: http://www. who.int/healthinfo/global_burden_disease/GBD_report_2004update_ full.pdf

3. Yusuf S, Sleight P, Pogue J, Bosch J, Davies R, Dagenais G. Effects of an angiotensin-converting-enzyme inhibitor, ramipril, on cardiovascular events in high-risk patients. The Heart Outcomes Prevention Evaluation Study Investigators. N Engl J Med. 2000;342(3):145-153.

4. Sleight P, Yusuf S, Pogue J, Tsuyuki R, et al. Blood-pressure reduction and cardiovascular risk in HOPE study. Lancet. 2001;358(9299):2130-2131.

5. Svensson P, de Faire U, Sleight $P$, et al. Comparative effects of ramipril on ambulatory and office blood pressures; A HOPE Substudy. Hypertension. 2000;38:e28-e32.

6. Freemantle N, Cleland J, Young P, et al. Beta blockade after myocardial infarction: systematic review and meta regression analysis. BMJ. 1999;318:1730-1737.

7. Ellison KE, Gandhi G. Optimizing the use of $\beta$-adrenoceptor antagonists in coronary artery disease. Drugs. 2005;65(6):787-797.

8. Antman EM, Hand M, Armstrong PW, et al. 2007 Focused Update of the ACC/AHA 2004 Guidelines for the Management of Patients With ST-Elevation Myocardial Infarction: A Report of the American College of Cardiology/American Heart Association Task Force on Practice Guidelines: Developed in Collaboration With the Canadian Cardiovascular Society Endorsed by the American Academy of Family Physicians: 2007 Writing Group to Review New Evidence and Update the ACC/AHA 2004 Guidelines for the Management of Patients With ST-Elevation Myocardial Infarction, Writing on Behalf of the 2004 Writing Committee. Circulation. 2008;117:296-329.

9. Kennedy H. Beta-blocker prevention of proarrhythmia and proischemia: clues from CAST, CAMIAT and EMIAT. Am J Cardiol. 1997;80(9):1208-1211.

10. Chobanian AV, Bakris GL, Black HR, et al. Seventh Report of the Joint National Committee on Prevention, Detection, Evaluation, and Treatment of High Blood Pressure. Hypertension. 2003; 42:1206-1252.

11. Mancia G, De Backer G, Dominiczak A, et al. The 2007 Guidelines for the Management of Arterial Hypertension. Eur Heart J. 2007;28:1462-1536.

12. Lindholm LH, Carlberg B, Samuelsson O. Should beta blockers remain first choice in the treatment of primary hypertension? A meta-analysis. Lancet. 2005;366(9496):1545-1553.

13. Khan N, McAlister FA. Re-examining the efficacy of beta-blockers for the treatment of hypertension: a meta-analysis. CMAJ. 2006;174(12):1737-1742.

14. The ALLHAT Officers and Coordinators for the ALLHAT Collaborative Research Group. Major outcomes in high-risk hypertensive patients randomized to angiotensin-converting enzyme inhibitor or calcium channel blocker vs diuretic: The Antihypertensive and Lipid-Lowering Treatment to Prevent Heart Attack Trial (ALLHAT). JAMA. 2002;288:2981-2997.

15. Rosendorff C, Black HR, Cannon CP, et al. AHA Scientific Statement: Treatment of hypertension in the prevention and management of ischemic heart disease.Circulation. 2007;115:2761-2788.

16. Croom KF, Wellington K. Modified-Release Nifedipine: A Review of the use of modified-release formulations in the treatment of hypertension and angina pectoris. Drugs. 2006;66(4):497-528.

17. Sorkin EM, Clissold SP, Brogden RN. Nifedipine: a review of its pharmacodynamic and pharmacokinetic properties, and therapeutic efficacy in ischaemic heart disease, hypertension, and related cardiovascular disorders. Drugs. 1985;30:182-274.
18. Grundy JS, Foster RT. The nifedipine gastrointestinal therapeutic system (GITS): evaluation of pharmaceutical, pharmacokinetic and pharmacological properties. Clin Pharmacokinet. 1996;30(1):28-51.

19. Heagerty AM. Nifedipine gastrointestinal therapeutic system hypertension management to improve cardiovascular outcomes. Int $J$ Clin Pract. 2005;(59)9:1112-1119.

20. Mancini GB. Antiatherosclerotic effects of calcium channel blockers. Prog Cardiovasc Dis. 2002;45:1-20.

21. Mancini GB, Miller ME, Evans GW; for PREVENT Study Group. Post hoc analysis of coronary findings from the Prospective Randomized Evaluation of the Vascular Effects of the Norvasc Trial (PREVENT). Am J Cardiol. 2002;89:1414-1416.

22. Simon A, Gariepy J, Moyse D, et al. Differential effects of nifedipine and co-amilozide on the progression of early carotid wall changes. Circulation. 2001;103:2949-2954.

23. Pitt B, Byington RP, Furberg CD, et al. Effect of amlodipine on the progression of atherosclerosis and the occurrence of clinical events. Circulation. 2000;102:1503-1510.

24. Zanchetti A, Bond MG, Hening M, et al. Calcium antagonist lacidipine slows down progression of asymptomatic carotid atherosclerosis. Circulation. 2002;106:2422-2427.

25. Psaty BM, Heckbert SR, Koepsell TD, et al. The risk of myocardial infarction associated with antihypertensive drug therapies. JAMA. 1995;274:620-625.

26. Furberg CD, Psaty BM, Meyer JV. Nifedipine: Dose-related increase in mortality in patients with coronary heart disease. Circulation. 1995;92:1326-1331.

27. Rafflenbeul W. Nifedipine in acute coronary syndromes: Furberg's refrain revisited. Eur Heart J. 1996;17:1147-1152.

28. Tijssen JGP, Hugenholtz PG. Critical appraisal of recent studies on nifedipine and other calcium channel blockers in coronary artery disease and hypertension. Eur Heart J. 1996;17:1152-1157.

29. Gong L, Zhang W, Zhu Y, et al. Shanghai Trial of Nifedipine in the Elderly (STONE). J Hypertens. 1996;14(10):1237-1245

30. Wu YK. Epidemiology and the community control of hypertension, stroke, and coronary heart disease in China. Chinese Med J. 1979;92:665-670.

31. Yui Y, Sumiyoshi T, Kodama K, et al. Comparison of nifedipine retard with angiotensin converting enzyme inhibitors in Japanese hypertensive patients with coronary artery disease: the Japan multicenter investigation for cardiovascular diseases-B (JMIC-B) randomized trial. Hypertens Res. 2004;27(3):181-191.

32. Pristipino C, Beltrame J, Finocchiaro ML, et al; Major racial differences in coronary constrictor response between Japanese and Caucasians with recent myocardial infarction. Circulation. 2000;101:1102-1108.

33. Japanese Society of Hypertension Guidelines Subcommittee for the Management of Hypertension: Guidelines for the management of hypertension for general practitioners. Hypertens Res. 2001; 24:613-634

34. Yoshitoshi Y, Kaneko Y, Saruta T. Antihypertensive effect of MK-421 (enalapril maleate) with concomitant thiazide diuretics in patients with essential hypertension: multiclinic pilot, open study. Jpn Pharmacol Ther. 1984;12:3439-3468.

35. Saruta T, Omae T, Iimura O, et al. Efficacy and safety of imidapril (ACE/TA-6366), angiotensin I converting enzyme inhibitor, in monotherapy on essential hypertension multiclinic open study. Rinsho Igaku.1991;7:2485-2504.

36. Arakawa K, Iimura O, Yoshinaga K, et al. Antihypertensive effect of lisinopril (MK-521) in essential hypertension: result of a multiclinic open trial. Saishin Igaku.1990;45:834-858.

37. Clinical Evaluation of Antihypertensive Drugs Group: Proposed guidelines for the clinical evaluation of antihypertensive drugs. Drug Res. 1979;10:849-864.

38. Shinoda E, Yoshiki Y, Kodama K, et al. Quantitative coronary angiogram analysis: nifedipine retard versus angiotensin-converting enzyme inhibitors (JMIC-B Side Arm Study). Hyertension. 2005;45:1153-1158. 
39. Brown MJ, Palmer CR, Castaigne A, et al. Morbidity and mortality in patients randomized to double-blind treatment with long-acting calcium-channel blocker or diuretic in the international nifedipine GITS Study: intervention as a goal in hypertension treatment (INSIGHT). Lancet. 2000;356:366-372.

40. Motro M, Shemesh J. Calcium channel blocker nifedipine slows down progression of coronary calcification in hypertensive patients compared with diuretics. Hypertension. 2001;37:1410-1413.

41. Poole-Wilson PA, Lubsen J, Kirwan BA, et al. Effect of long-acting nifedipine on mortality and cardiovascular morbidity in patients with stable angina requiring treatment (ACTION trial): randomized controlled trial. Lancet. 2004;364:849-857.

42. Pitt B, Byington RP, Furberg CD, et al. Effect of amlodipine on the progression of atherosclerosis and the occurrence of clinical events. Circulation. 2000;102(13):1503-1510.

43. Ozaki Y, Keane D, Serruys PW. Progression and regression of coronary stenosis in the long-term follow-up of vasospastic angina. Circulation. 1995;92:2446-2456.

44. Dens JA, Desmet WJ, Coussement P, et al. Long term effects of nisoldipine on the progression of coronary atherosclerosis and the occurrence of clinical events: the NICOLE study. Heart. 2003;89:887-892.

45. Nissen SE, Tuzcu EM, Libby P, et al. Effect of antihypertensive agents on cardiovascular events in patients with coronary disease and normal blood pressure. The CAMELOT study: a randomized controlled trial. JAMA. 2004;292:2217-2226.

46. Tatti P, Pahor M, Byington RP, et al. Outcome results of the fosinopril versus amlodipine cardiovascular events randomized trial (FACET) in patients with hypertension and NIDDM. Diabetes Care. 1998;21(4):597-603.

47. Fortini A, Cappelletti C, Cecchi L, Laureano R. Fosinopril in the treatment of hypertension: effects on $24 \mathrm{~h}$ ambulatory blood pressure and on blood pressure response to exercise. J Hum Hypertens. 1994;8:469-474.

48. Bainbridge AD, Herlihy O, Meredith, PA, Elliott HL. A comparative assessment of amlodipine and felodipine ER: pharmacokinetic and pharmacodynamic indices. Eur J Clin Pharmacol. 1993; 45:425-430.

49. Kirkendall WM. Comparative assessment of first-line agents for treatment of hypertension. Am J Med. 1988;84(Suppl 3B):32-41.

50. Weinstein DB, Heider JG. Antiatherogenic properties of calcium antagonists. Am J Cardiol. 1987;59:163B-172B.

51. Chobanian AV. Effects of calcium antagonists and other antihypertensive drugs on atherosclerosis. J Hypertens Suppl. 1987;5(Suppl 4):43-48.

52. Weinstein DB, Heider JG. Protective action of calcium antagonists in atherosclerosis and experimental vascular injury. Am J Hypertens. 1989;2:205-212.

53. Bond MG, Purvis C, Mercuri M. Antiatherogenic properties of calcium antagonists. J Cardiovasc Pharmacol. 1991;17(Supp1 4):87-93.
54. Borhani No, Mercuri M, Borhani PA, et al. Final outcome results of the multicenter isradipine diuretic atherosclerosis study (MIDAS): A randomized controlled trial. JAMA. 1996;276(10):785-791.

55. Furberg CD, Byington RP, Borhani NO. Design features: multicenter isradipine diuretic atherosclerosis Study (MIDAS). Am J Med. 1989;86(Suppl 4A):37-39.

56. Borhani NO, Bond MG, Sowers JR, et al. The MIDAS: a study of the antiatherogenic properties of isradipine in hypertensive patients. J Cardiovasc Pharmacol. 1991;18(Suppl 3):15-19.

57. Hansson L, Lindholm LH, Ekbom T, et al. Randomised trial of old and new antihypertensive drugs in elderly patients: cardiovascular mortality and morbidity the Swedish Trial in Old Patients with Hypertension-2 study (STOP-2). Lancet. 1999;354:1751-1756.

58. Dahlof B, Sever PS, Poulter NR, et al. Prevention of cardiovascular events with an antihypertensive regimen of amlodipine adding perindopril as required versus atenolol adding bendroflumethiazide as required, in the Anglo-Scandinavian cardiac outcomes trial-blood pressure lowering arm (ASCOT-BPLA): a multicentre randomized controlled trial. The Lancet. 2005;366 (9489):895-906

59. Staessen JA, Fagard E, Thijs L, et al. Randomized double-blind comparison of placebo and active treatment for older patients with isolated systolic hypertension. Lancet. 1997;350:757-764.

60. National Intervention Cooperative Study in Elderly Hypertensives Study Group. Randomized double-blind comparison of a calcium antagonist and a diuretic in elderly hypertensives. Hypertension. 1999;34:1129-1133.

61. Dahlof A, Lindholm LH, Hansson L, et al. Morbidity and mortality in the Swedish trial in old Patients with hypertension (STOP-Hypertension). Lancet. 1991;338:1281-1285.

62. Savage PJ, Pressel SL, Curb JD; for the SHEP Cooperative Research Group. Influence of long-term, low-dose, diuretic-based, antihypertensive therapy on glucose, lipid, uric acid, and potassium levels in older men and women with isolated systolic hypertension: the Systolic Hypertension in the Elderly Program. Arch Intern Med. 1998;158:741-751.

63. MRC Working Party. Medical Research Council trial of treatment of hypertension in older adults: principal results. $B M J$. 1992;304:405-412.

64. Liu L, Wang G, Gong L, Liu G, Staessen JA. Comparison of active treatment and placebo in older Chinese patients with isolated systolic hypertension. J Hypertens. 1998;16:1823-1829.

65. Jamerson K, Weber MA, Bakris GL, et al. Benazepril plus amlodipine or hydrochlorothiazide for hypertension in high-risk patients. $N$ Engl J Med. 2008;359(23):2417-2428

66. Chobanian AV. Does It Matter How Hypertension Is Controlled? N Engl J Med. 2008;359(23):2485-2487.

67. Hosoda S, Iino T, Yasuda H, et al. Long-term follow-up of medically treated patients with coronary artery disease: incidence of major cardiac events and its risk factors in Japanese with coronary artery disease. Jpn Circ J. 1990;54:231-240.
Vascular Health and Risk Management

\section{Publish your work in this journal}

Vascular Health and Risk Management is an international, peerreviewed journal of therapeutics and risk management, focusing on concise rapid reporting of clinical studies on the processes involved in the maintenance of vascular health; the monitoring, prevention and treatment of vascular disease and its sequelae; and the involvement of

\section{Dovepress}

metabolic disorders, particularly diabetes. This journal is indexed on PubMed Central and MedLine. The manuscript management system is completely online and includes a very quick and fair peer-review system, which is all easy to use. Visit http://www.dovepress.com/ testimonials.php to read real quotes from published authors. 